\title{
Mathematical modelling of COVID-19 transmission and control strategies in the population of Bauchi State, Nigeria
}

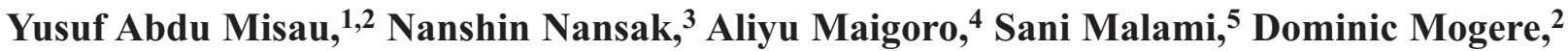 \\ Suleiman Mbaruk, ${ }^{2}$ Rilwanu Mohammed, ${ }^{4}$ Suleiman Lawal, ${ }^{6}$ Sunusi Usman Usman ${ }^{7}$
}

${ }^{1}$ Department of Community Medicine, College of Medical Sciences Abubakar Tafawa Balewa University, Bauchi State, Nigeria; ${ }^{2}$ Department of Epidemiology and Biostatistics, School of Public Health Mount Kenya University, Thika, Kenya; ${ }^{3}$ Zonal Advance Space Applications Laboratory Kashere, Gombe State, Nigeria; ${ }^{4}$ Ministry of Health, Bauchi State, Nigeria; ${ }^{5}$ Department of Histopathology, College of Medical Sciences, Abubakar Tafawa Balewa University Teaching Hospital, Bauchi State, Nigeria; ${ }^{6}$ Depatment of Medicine, Abubakar Tafawa Balewa University Teaching Hospital, Bauchi State, Nigeria; ${ }^{7}$ Department of Public Health, Bauchi State University, Gadau, Bauchi State, Nigeria

\begin{abstract}
The novel SARS-COV-2 has since been declared a pandemic by the World Health Organization (WHO). The virus has spread from Wuhan city in China in December 2019 to no fewer than 200 countries as at June 2020 and still counting. Nigeria is currently experiencing a rapid spread of the virus amidst weak health system
\end{abstract}

Correspondence: Yusuf Abdu Misau, Department of Community Medicine, College of Medical Sciences, Abubakar Tafawa Balewa University Bauchi, Nigeria

E-mail: yusufmisau@yahoo.com

Key words: COVID-19, Modelling, Bauchi, Nigeria

Contributions: YAM: Conceptualized research topic and developed proposal; NN: Statistical analysis; AM: Literature review; RM: Methods design; SL: Results reports writing; SUU: Draft discussions and conclusion; SM, DM and SM: Review of draft manuscript.

Conflict of interest: The authors declare no potential conflict of interest.

Funding: This study is partly funded by Bauchi state Ministry of health, COVID-19 Rapid Response Committee.

Availability of data and materials: This study utilizes publicly available data from Bauchi state Ministry of health via SORMAS platform.

Ethics approval: Ethical approval for this study was granted by Bauchi state health research ethics committee via approval letter: Protocol Reg No. BSMOH/REC/27/2020; Approval: NREC/03/11/19B/2020/30.

Received for publication: 30 June 2020.

Revision received: 13 July 2020.

Accepted for publication: 13 July 2020.

This work is licensed under a Creative Commons Attribution NonCommercial 4.0 License (CC BY-NC 4.0).

(C) Copyright: the Author(s),2020

Licensee PAGEPress, Italy

Annals of African Medical Research 2020; 3:120

doi:10.4081/aamr.2020.120 and more than $80 \%$ of population leaving on less than 1USD per day. To help understand the behavior of the virus in resource limited settings, we modelled the outbreak of COVID-19 and effects of control strategies in Bauchi state at north-eastern Nigeria. Using the real data of Bauchi state COVID-19 project, this research work extends the epidemic SEIR model by introducing new parameters based on the transmission dynamics of the novel COVID-19 pandemic and preventive measures. The total population of Bauchi State at the time of the study, given by is compartmentalized into five (5) different compartments as follows: Susceptible $(S)$, Exposed (E), Infectious (I), Quarantined (Q) and Recovered (R). The new model is SEIQR. $N=S \rightarrow E \rightarrow I \rightarrow Q \rightarrow R$

Data was collected by accessing Bauchi state electronic database of COVID-19 project to derive all the model parameters, while analysis and model building was done using Maple software. At the time of this study, it was found that the reproduction number $\mathrm{R}$, for COVID-19 in Bauchi state, is $2.6 \times 10^{-5}$. The reproduction number $\mathrm{R}$ decreased due to the application of control measures. The compartmental SEIRQ model in this study, which is a deterministic system of linear differential equations, has a continuum of disease-free equilibria, which is rigorously shown to be locallyasymptotically stable as the epidemiological threshold, known as the control reproduction number $\mathrm{R}=0.0000026$ is less than unity. The implication of this study is that the COVID-19 pandemic can be effectively controlled in Bauchi, since is $\mathrm{R}<1$. Contact tracing and isolation must be increased as the models shows, the rise in infected class is a sign of high vulnerability of the population. Unless control measures are stepped up, despite high rate of recovery as shown by this study, infection rate will keep increasing as currently there is a no vaccine for COVID-19.

\section{Introduction}

Evidence has shown that the "speed and scope of detection of an infectious disease, in particular, timely identification and reporting of a new pathogen, is a major indicator of a country's ability to control infectious diseases. Furthermore, the Global Health Security (GHS) index shows that only $19 \%$ of countries have the ability to quickly detect and report epidemics of potential international concern, and fewer than $5 \%$ of countries can rapidly respond to and mitigate the spread of an epidemic, and no country is fully prepared for epidemics or pandemics just as the coronavirus disease 2019 (COVID-19) seems to have confirmed". ${ }^{1}$ The COVID- 
19 caused by severe acute respiratory syndrome coronavirus 2 (SARS-CoV-2), which originated in Wuhan city, Hubei province, in December, $2019,{ }^{2}$ has already been declared pandemic by the World Health Organization (WHO) on $11^{\text {th }}$ March $2020 .^{3}$ The disease has myriad of systemic and respiratory symptoms some cases, deaths especially among elderly or co-morbid positive patients as hallmarks. ${ }^{4}$ The rapid spread of COVID-19 through respiratory secretions aerosol, droplets on hands or airborne transmission permits its ubiquity and made it the biggest global public health challenge since the plagues of $19^{\text {th }}$ century. ${ }^{5}$

As at $17^{\text {th }}$ May 2020 , there are $4,756,426$ confirmed cases globally about $2,609,449$ had their case outcomes closed. Of these, $313,940(15 \%)$ were deaths and $1,833,037(85 \%)$ recovered cases. ${ }^{6}$ Nigeria accounts for 5,621 confirmed cases, 176 deaths and 1,472 recoveries of the global burden for the same duration. ${ }^{7}$

Bauchi state in Northern Nigeria, is a major agricultural hub of the country. It plays unique and strategic roles in the socio-geopolitical affairs of the country's Northeast region as well. About 222 confirmed cases, 5 deaths and 103 recoveries of COVID-19 have already been documented. ${ }^{8}$ Bauchi state, like other 36 Nigeria's states has implemented measures such as closing of schools and universities, the shutdown sports centers, suspension of weekly Mosques and Church services, cultural and social gatherings, closing of markets periodically, implementation of a curfew from 8PM to 6AM seven days a week, the prohibition of public gatherings containing more than 50 people, cutting down carder of public workers reporting for work, closing of inter-state boarders and limiting number of passengers allowable on commercial buses during intra-state travels. To what extent, these measures are helping the control of COVID-19 spread in the state is not precisely known.

"Driven by a disease's intrinsic mechanism or fitted through sufficient data, mathematical models have been used to simulate scenarios and predict evolution of infectious diseases since the early 20th century". ${ }^{1}$ Expectedly, models will serve as means to provide quick insights of, and predictive power on, a new pathogen in the early stages of an outbreak, which are seemingly contradictory expectations though. ${ }^{1}$ Regardless, models provide stakeholders opportunity to plan resources for controlling outbreaks especially in resource limited settings. Our aim in this study is to simulate COVID-19 transmission dynamics in the population of Bauchi state Nigeria and determine the effects of control measures in slowing the rate of the transmission.

\section{Materials and Methods}

\section{Model formulation}

This research work extends the epidemic SEIR model by introducing new parameters based on the transmission dynamics of the novel covid-19 pandemic and preventive measures.

The total population of Bauchi State at the time of the study, given by $N(t)$ is compartmentalized into five (5) different compartments as follows: Susceptible $(S)$, Exposed $(E)$, Infectious $(I)$, Quarantined $(Q)$ and Recovered $(R)$. The new model is SEIQR (Figure 1).

$N=S \rightarrow E \rightarrow I \rightarrow Q \rightarrow R$

\section{Assumption}

$\alpha=$ Rate of death cause by covid-19

$\varepsilon=$ rate of recovery $\gamma=$ rate of transmission

$\beta=$ recruitment rate

$d=$ Natural death

$m=$ Rate of Quarantine

$\delta=$ coronavirus incubation period

$\tau=$ Treatment rate

$\varphi(\mathrm{t})=$ incidence rate

Assumptions:

I. It is assumed that with the interstate lockdown, Bauchi state population remains constant throughout the time of this research.

II. Infected class move into quarantined class and possibly Recovered Class.

III. It is assumed that mobility from Susceptible population to Exposed class occurs only when susceptible gets in contact with infected population.

IV. It is assumed that every Exposed and infected class exhibits Covid-19 symptoms within 14 days

V. Death due to covid-19 occurs at the rate $\alpha$

VI. Recruitment from susceptible to infected is through contact.

\section{The SEIQR model formulation and method}

$$
\begin{aligned}
& \frac{d S(t)}{d t}=\beta-\gamma S I-\alpha S \\
& \frac{d E(t)}{d t}=\gamma S I-(\alpha+\delta) E \\
& \frac{d I(t)}{d t}=\delta E-(\alpha+\tau+d) I \\
& \frac{d R(t)}{d t}=\delta E-(\varepsilon+d) R \\
& \frac{d Q(t)}{d t}=\varepsilon R-(d+m) Q
\end{aligned}
$$

The force of infection in Bauchi State is given by the fraction

$\Psi(t)=\frac{\not I+\tau R+\varepsilon Q}{N}$

Therefore

$$
\begin{aligned}
& S=S(t), E=E(t), I=I(t), Q=Q(t), R=R(t) \text { thus } \\
& N(t)=S(t)+E(t)+I(t)+Q(t)+R(t)
\end{aligned}
$$

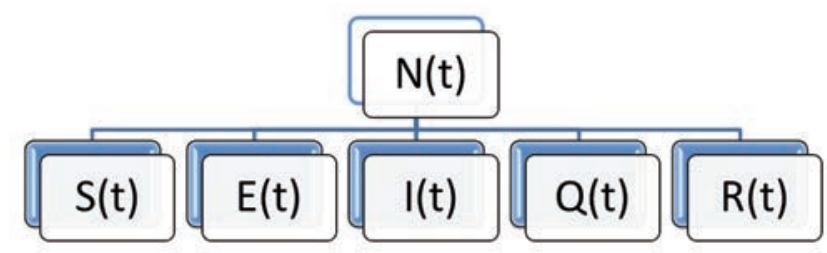

Figure 1. The new SEIQR model. 
By differentiating $N(t)$ with respect to time $(t)$, we obtain:

$$
\begin{aligned}
& \frac{d N(t)}{d t}=\frac{d S(t)}{d t}+\frac{d E(t)}{d t}+\frac{d I(t)}{d t}+\frac{d Q(t)}{d t}+\frac{d R(t)}{d t} \\
& \frac{d N(t)}{d t}=\beta-\gamma \delta I-\alpha S+\gamma S I-(\alpha+\delta) E+\delta E-(\alpha+\tau+d) I+\delta E-(\varepsilon+d) R+\varepsilon R-(d+m) Q \\
& \frac{d N(t)}{d t}=\beta-\gamma S I-\alpha S+\gamma S I-\alpha E-\delta E+\delta E-\alpha I-\tau I-d I+\delta E-\varepsilon R-d R+\varepsilon R-d Q-m Q \\
& \frac{d N(t)}{d t}=-\gamma S I+\gamma S I-\delta E+\delta E-\varepsilon R+\varepsilon R-\alpha S-\alpha E-\alpha I-\tau I-d I+\delta E-d R-d Q-m Q+\beta \\
& \frac{d N(t)}{d t}=\beta-\alpha S-\alpha E-\alpha I-\tau I-d I+\delta E-d R-d Q-m Q \\
& \frac{d N(t)}{d t}=\beta-\alpha S-(\alpha-\delta) E-(\alpha+\tau+d) I-d R-(d+m) Q
\end{aligned}
$$

Treating the equation as a vector, each derivative can be normalized as follows (Victor 2020):

$s=\frac{S}{N}, e=\frac{E}{N}, i=\frac{I}{N} r=\frac{R}{N}, q=\frac{Q}{N}$

$\frac{d s}{d t}=\frac{1}{N}\left[\frac{d S(t)}{d t}-s \frac{d N(t)}{d t}\right]$

Substituting equations (1) and (11) into (13) gives the following:

$$
\begin{aligned}
& \frac{d s}{d t}=\frac{1}{N}[\beta-\gamma \delta I-\alpha S-s(\beta-\alpha S-(\alpha-\delta) E-(\alpha+\tau+d) I-d R-(d+m) Q)] \\
& \frac{d s}{d t}=\frac{1}{N}[\beta-\gamma \delta I-\alpha S-(-s \beta-\alpha s S-(s \alpha-s \delta) E-(s \alpha+s \tau+s d) I-s d R-(s d+s m) Q)] \\
& =\alpha s^{2}-\alpha s-\beta s+\beta-\frac{\gamma \delta i}{N}+(\alpha-\delta) s e+(\alpha+\tau+d) s i+d s r+(d+m) s q \\
& \text { (1ד) }
\end{aligned}
$$

In the same way,

$$
\begin{aligned}
& \frac{d e}{d t}=\frac{1}{N}\left[\frac{d E(t)}{d t}-e \frac{d N(t)}{d t}\right] \\
& \frac{d e}{d t}=\frac{1}{N}[\delta \delta-(\alpha+\delta) E-e(\beta-\alpha S-(\alpha-\delta) E-(\alpha+\tau+d) l-d R-(d+m) Q)] \\
& \frac{d e}{d t}=\frac{1}{N}[s i-(\alpha+\delta) e-e(\beta-\alpha s-(\alpha-\delta) e-(\alpha+\tau+d) i-d r-(d+m) q)] \\
& \left.\frac{d e}{d t}=\left[(\alpha-\delta) e^{2}-(\alpha+\delta) e-\beta e+\alpha s e+\frac{\gamma \delta}{N}+(\alpha+\tau+d) e i+d r e+(d+m) q e\right)\right]
\end{aligned}
$$

Again we have

$$
\begin{aligned}
\frac{d i}{d t}= & \frac{1}{N}[\delta E-(\alpha+\tau+d) I-i(\beta-\alpha S-(\alpha-\delta) E-(\alpha+\tau+d) I-d R-(d+m) Q)] \\
& \left.\frac{d i}{d t}=\left[(\alpha+\tau+d) i^{2}+(\alpha+\tau+d) i-\beta i+\delta e-\frac{\alpha s i}{N}+(\alpha-\delta) i e+d r i+(d+m) q i\right)\right] \\
\frac{d r}{d t}= & \frac{1}{N}\left[\frac{d R(t)}{d t}-r \frac{d N(t)}{d t}\right] \\
\frac{d r}{d t}= & \frac{1}{N}[\delta E-(\delta+d) R-r(\beta-\alpha S-(\alpha-\delta) E-(\alpha+\tau+d) I-d R-(d+m) Q)] \\
\frac{d r}{d t}= & d r^{2}-(\varepsilon+d) r-\beta r+\delta e+\frac{\alpha r s}{N}+(\alpha-\delta) r e+(\alpha+\tau+d) r i-(d+m) q r
\end{aligned}
$$

Lastly, we can normalize the quarantined derivative

$$
\begin{aligned}
& \frac{d q}{d t}=\frac{1}{N}[\varepsilon R-(d+m) Q-q(\beta-\alpha S-(\alpha-\delta) E-(\alpha+\tau+d) I-d R-(d+m) Q)] \\
& \left.\left[a r-(d+m) q-\beta q+\frac{\alpha q s}{N}+(\alpha-\delta) e q+(\alpha+\tau+d) i q+d r q+(d+m) q^{2}\right)\right] \\
& \left.=\left[(d+m) q^{2}-(d+m) q-\beta q+\sigma+\frac{\alpha q s}{N}+(\alpha-\delta) e q+(\alpha+\tau+d) i q+d r q\right)\right]
\end{aligned}
$$

Most importantly

$s+e+I+q+r=1$

\section{Data Source and Statistical Analysis}

The below system of differential equation is the formulated model which can be solved using Runge-Kutta method because of its apparent complexity. ${ }^{9}$ Each of the variable and parameter has its meaning and values shall be assigned to each variable as it will be obtained from the Bauchi state ministry of health and WHO.

\section{The SEIQR mathematical model}

$$
\begin{aligned}
& \frac{d s}{d t}=\alpha s^{2}-\alpha s-\beta s+\beta-\frac{\gamma s i}{N}+(\alpha-\delta) s e+(\alpha+\tau+d) s i+d s r+(d+m) s q \\
& \frac{d e}{d t}=(\alpha-\delta) e^{2}-(\alpha+\delta) e-\beta e+\alpha s e+\frac{\gamma s i}{N}+(\alpha+\tau+d) e i+d r e+(d+m) q e \\
& \frac{d i}{d t}=(\alpha+\tau+d) i^{2}+(\alpha+\tau+d) i-\beta i+\delta e-\frac{\alpha s i}{N}+(\alpha-\delta) i e+d r i+(d+m) q i \\
& \frac{d r}{d t}=d r^{2}-(\varepsilon+d) r-\beta r+\delta e+\frac{\alpha r s}{N}+(\alpha-\delta) r e+(\alpha+\tau+d) r i-(d+m) q r \\
& \frac{d q}{d t}=(d+m) q^{2}-(d+m) q-\beta q+\varepsilon r+\frac{\alpha q s}{N}+(\alpha-\delta) e q+(\alpha+\tau+d) i q+d r q
\end{aligned}
$$

\section{Disease free equilibrium state of SEIQR model}

At equilibrium state, every component of the SEIQR model is set to zero. That is to say, $s=e=i=q=r=0$; also, the derivatives of the disease component turn zero. By truncating equation (14) based on the descending order of the compsonent susceptible class $(s)$, we get

$$
\begin{gathered}
\alpha s^{2}-\alpha s-\beta s-\frac{\gamma s i}{N} \ldots=0 \\
\frac{\gamma s i}{N} \ldots=0
\end{gathered}
$$

setting

we have $\alpha s^{2}-\alpha s-\beta s=0$

this gives an equation in the form of $\alpha x^{2}+b x+c=0$ which is quadratic.

where $\mathrm{A}=\alpha, B=-\alpha ; C=-\beta$ by quadratic formula, we obtain

$s=\frac{\alpha \pm \sqrt{\alpha^{2}+4 \alpha \beta}}{2 \alpha}$

The solution of the quadratic equation above can never be imaginary or negative. It will always be positive since the parameter ( Rate of death caused by covid-19) can only take zero when no one dies. Same goes for (recruitment rate). i.e $\left(s_{1}, s_{2}\right)=(0,=)$ or $\left(s_{1}>0, s_{1}>0\right)$. This solution holds good in $\alpha s^{2}-\alpha s-\beta s=0$. 


\section{Stability analysis of disease-free equilibrium state}

It has been established that all components are zero at this state i.e $E_{o}=(S, E, I, R, Q)=(0,0,0,0,0)$ By linearizing the system of differential equation, (proposed model), given by:

$$
\begin{aligned}
& f_{s}=\alpha s^{2}-\alpha s-\beta s+\beta-\frac{\gamma \delta i}{N}+(\alpha-\delta) s e+(\alpha+\tau+d) s i+d s r+(d+m) s q \\
& f_{e}=(\alpha-\delta) e^{2}-(\alpha+\delta) e-\beta e+\alpha s e+\frac{\gamma s i}{N}+(\alpha+\tau+d) e i+d r e+(d+m) q e \\
& f_{i}=(\alpha+\tau+d) i^{2}+(\alpha+\tau+d) i-\beta i+\delta e-\frac{\alpha s i}{N}+(\alpha-\delta) i e+d r i+(d+m) q i \\
& f_{r}=d r^{2}-(\varepsilon+d) r-\beta r+\delta e+\frac{\alpha r s}{N}+(\alpha-\delta) r e+(\alpha+\tau+d) r i-(d+m) q r \\
& f_{q}=(d+m) q^{2}-(d+m) q-\beta q+\varepsilon r+\frac{\alpha q s}{N}+(\alpha-\delta) e q+(\alpha+\tau+d) i q+d r q
\end{aligned}
$$

Differentiating each model partially gives

$$
\begin{aligned}
& \frac{\partial f_{s}}{\partial s}=-(\alpha+\beta), \frac{\partial f_{s}}{\partial e}=0, \frac{\partial f_{s}}{\partial i}=0, \frac{\partial f_{s}}{\partial r}=0, \frac{\partial f_{s}}{\partial q}=0 \\
& \frac{\partial f_{s}}{\partial s}=0, \frac{\partial f_{e}}{\partial e}=-(\alpha+\delta), \frac{\partial f_{r}}{\partial i}=0, \frac{\partial f_{e}}{\partial r}=0, \frac{\partial f_{c}}{\partial q}=0 \\
& \frac{\partial f_{i}}{\partial s}=0, \frac{\partial f_{i}}{\partial e}=\delta, \frac{\partial f_{i}}{\partial i}=(\alpha+\tau+d)-\beta, \frac{\partial f_{i}}{\partial r}=0, \frac{\partial f_{i}}{\partial q}=0 \\
& \frac{\partial f_{r}}{\partial s}=0, \frac{\partial f_{r}}{\partial e}=\delta, \frac{\partial f_{r}}{\partial i}=0, \frac{\partial f_{r}}{\partial r}=-(\varepsilon+\delta)-\beta, \frac{\partial f_{r}}{\partial q}=0 \\
& \frac{\partial f_{q}}{\partial s}=0, \frac{\partial f_{q}}{\partial e}=0, \frac{\partial f_{q}}{\partial i}=0, \frac{\partial f_{q}}{\partial r}=\varepsilon, \frac{\partial f_{q}}{\partial q}=-(d+m)-\beta
\end{aligned}
$$

This can be written in matrix form with Jacobian $J$ thus

$$
\begin{aligned}
& J=\left[\begin{array}{ccccc}
-(\alpha+\beta) & 0 & 0 & 0 & 0 \\
0 & -(\alpha+\delta) & 0 & 0 & 0 \\
0 & \delta & (\alpha+\tau+d)-\beta & 0 & 0 \\
0 & \delta & 0 & -(\varepsilon+\delta)-\beta & 0 \\
0 & 0 & 0 & \varepsilon & -(d+m)-\beta
\end{array}\right] \\
& \operatorname{Det}(J)=0=\left|\begin{array}{ccccc}
-(\alpha+\beta) & 0 & 0 & 0 & 0 \\
0 & -(\alpha+\delta) & 0 & 0 & 0 \\
0 & \delta & (\alpha+\tau+d)-\beta & 0 & 0 \\
0 & \delta & 0 & -(\varepsilon+\delta)-\beta & 0 \\
0 & 0 & 0 & \varepsilon & -(d+m)-\beta
\end{array}\right|=0
\end{aligned}
$$

i.e $\operatorname{Det}(J)=0$ and the trace of $J$ written as $\operatorname{Trace}(J)$ i.e the sum of the elements in the leading diagonal given as:

$$
\operatorname{Trace}(J)=-(\alpha+\beta)-(\alpha+\delta)+(\alpha+\tau+d)-\beta-(\varepsilon+\delta)-\beta-(d+m)-\beta>0
$$

\section{Basic reproductive number of the model}

The basic Reproductive Number denoted by $R_{o}$ is the number of cases that are expected to occur on average in a homogeneous population as a result of infection by a single individual, when the population is susceptible at the beginning of an epidemic, before widespread immunity starts to develop and before any attempt is made at vaccination. ${ }^{10}$ If

I. $\quad R_{o}>1$ This means infection will spread exponentially

II. $R_{o}<1$ Infection will spread slowly and eventually die out III. $R_{o}=1$ Is the threshold number. gies.

The values of $R_{o}$ will guide on prevention and control strate-
$F_{i}=\left(\begin{array}{l}\varphi e+d i \\ \varphi e\end{array}\right)$

By linearization and obtaining of partial derivatives of $V_{i}$ at disease free equilibrium $\left(E_{o}\right)$.

$$
V=\left[\frac{\partial V_{i}}{\partial x_{i}}\left(E_{o}\right)\right]=\left(\begin{array}{cc}
-(\tau+\alpha) & 0 \\
0 & -(\tau+\alpha)
\end{array}\right)
$$

with $l \leq i, j \geq m, m$ is the number of the class of people infected with Covid-19 in Bauchi State.

Suppose $m=2$ then it suffices to say

$$
\begin{aligned}
& F=\left(\begin{array}{ll}
\varphi & d \\
0 & \varphi
\end{array}\right) \\
& V^{-1}=\left(\begin{array}{cc}
-\frac{1}{(\tau+\alpha)} & 0 \\
0 & -\frac{1}{(\tau+\alpha)}
\end{array}\right) F V^{-1}=\left(\begin{array}{ll}
\varphi & d \\
0 & \varphi
\end{array}\right)\left(\begin{array}{cc}
-\frac{1}{(\tau+\alpha)} & 0 \\
0 & -\frac{1}{(\tau+\alpha)}
\end{array}\right)=\left(\begin{array}{cc}
-\frac{\varphi}{(\tau+\alpha)} & 0 \\
0 & -\frac{\varphi}{(\tau+\alpha)}
\end{array}\right)
\end{aligned}
$$

Now by obtaining the characteristics polynomial and solving for the eigenvalues, we have

i.e

$$
\begin{aligned}
& \left|F V^{-1}-\lambda I\right|=\mathbf{0}_{\text {i.e }}\left(\begin{array}{cc}
-\frac{\varphi}{(\tau+\alpha)} & 0 \\
0 & -\frac{\varphi}{(\tau+\alpha)}
\end{array}\right)-\lambda\left(\begin{array}{ll}
1 & 0 \\
0 & 1
\end{array}\right)=0 \\
& \left|F V^{-1}-\lambda\right|=\left(\begin{array}{cc}
-\frac{\varphi}{(\tau+\alpha)} & 0 \\
0 & -\frac{\varphi}{(\tau+\alpha)}
\end{array}\right)-\left(\begin{array}{ll}
\lambda & 0 \\
0 & \lambda
\end{array}\right)=\left(\begin{array}{cc}
-\frac{\varphi}{(\tau+\alpha)}-\lambda & 0 \\
0 & -\frac{\varphi}{(\tau+\alpha)}-\lambda
\end{array}\right)=0 \\
& \left|F V^{-1}-\lambda I\right|=\left|\begin{array}{cc}
-\frac{\varphi}{(\tau+\alpha)}-\lambda & 0 \\
0 & -\frac{\varphi}{(\tau+\alpha)}-\lambda
\end{array}\right|=0 \\
& \lambda^{2}+\left(\frac{2 \varphi}{\tau+\alpha}\right) \lambda+\left(\frac{\varphi}{\tau+\alpha}\right)^{2}=0
\end{aligned}
$$

This is quadratic.

There are two eigenvalues $\lambda_{1}, \lambda_{2}$ corresponding to the roots of the characteristic's polynomial, the dominant eigenvalue is considered the basic reproductive number $R_{o}$. So, we have from the solution that 


$$
R_{o}=-\left(\frac{\varphi}{\tau+\alpha}\right)<1
$$

The Basic Reproductive Number $R_{o}$. given by $R_{o}=-\left(\frac{\varphi}{\tau+\alpha}\right)<1$ means there will be a decline in secondary infections in Bauchi state if all precautionary measures( most especially quarantine) are strictly adhered to in Bauchi. This is apparent since the ratio of incidence rate to the infected class is negative.

\section{Ethical considerations}

In order to ensure strict compliance with the international standard for conduct of human research, this study was subjected to and granted ethical approval by Bauchi state health ethics research committee. Since the study used secondary data, we ensured strict confidentiality by removing names and any identifier before data analysis.

\section{Results}

At Diseases Free equilibrium, $S=E=I=Q=R=0$, (that is to say at time $\mathrm{t}=0$, the various population classes are zero.

$$
\begin{aligned}
& r_{o}=\frac{R_{o}}{N}=\frac{0}{7,468,016}=0, \\
& i_{o}=\frac{I_{o}}{N}=\frac{0}{7,468,016}=0, \\
& e_{o}=\frac{E_{o}}{N}=\frac{0}{7,468,016}=0, \\
& s_{o}=\frac{S_{o}}{N}=\frac{0}{7,468,016}=0 \\
& \text { and } q_{o}=\frac{Q_{o}}{N}=\frac{0}{7,468,016}=0
\end{aligned}
$$

and

showing $s_{o}=e_{o}=i_{o}=q_{o}=r_{o}=0$

\section{Data}

Data are presented in Table 1.

\section{Susceptible class model solution}

$$
\begin{gathered}
\frac{d s}{d t}=\alpha s^{2}-\alpha s-\beta s+\beta-\frac{\gamma s i}{N}+(\alpha-\delta) s e+(\alpha+\tau+d) s i+d s r+(d+m) s q \\
\int \frac{d s}{d t} d t=\int\left[\alpha s^{2}-\alpha s-\beta s+\beta-\frac{\gamma \delta i}{N}+(\alpha-\delta) s e+(\alpha+\tau+d) s i+d s r+(d+m) s q\right] d t \\
s(t)=\alpha s^{2} t-\alpha s t-\beta s t+\beta t-\frac{\gamma s i}{N} t+(\alpha-\delta) s e t+(\alpha+\tau+d) s i t+d s t t+(d+m) s q t+c(t) \\
s(0)=c=0 \Rightarrow c=0
\end{gathered}
$$

Equation (21) is the solution of the susceptible class model and the values of all the variables are already known. What changes however is time which runs from 0 to 14. i.e. $(0 \leq t \leq 14)$. 14 is not taken arbitrary as the upper boundary of the interval, it is taken because the virus is said to manifest within 14 days. For every value of $t$, there is a corresponding value of $s(t)$ (Figure 2).

\section{Exposed class model solution}

$$
\begin{gathered}
\frac{d e}{d t}=(\alpha-\delta) e^{2}-(\alpha+\delta) e-\beta e+\alpha s e+\frac{\gamma i}{N}+(\alpha+\tau+d) e i+d r e+(d+m) q e \\
\int \frac{d e}{d t} d t=\int\left[(\alpha-\delta) e^{2}-(\alpha+\delta) e-\beta e+\alpha s e+\frac{\gamma \delta i}{N}+(\alpha+\tau+d) e i+d r e+(d+m) q e\right] d t \\
e(t)=(\alpha-\delta) e^{2} t-(\alpha+\delta) e t-\beta e t+\alpha s e t+\frac{\gamma \delta i}{N} t+(\alpha+\tau+d) e i t+d r e t+(d+m) q e t+k(t)
\end{gathered}
$$

$e(0)=k=0$ at time $t=0$

\section{Infected class model solution}

$$
\begin{gathered}
\frac{d i}{d t}=(\alpha+\tau+d) i^{2}+(\alpha+\tau+d) i-\beta i+\delta e-\frac{\alpha s i}{N}+(\alpha-\delta) i e+d r i+(d+m) q i \\
\int \frac{d i}{d t} d t=\int\left[(\alpha+\tau+d) i^{2}+(\alpha+\tau+d) i-\beta i+\delta e-\frac{\alpha s i}{N}+(\alpha-\delta) i e+d r i+(d+m) q i\right) d t \\
i(t)=(\alpha+\tau+d) i^{2} t+(\alpha+\tau+d) i t-\beta i t+\delta e t-\frac{\alpha s i}{N} t+(\alpha-\delta) i e t+d r i t+(d+m) q i t+u(t) \\
i(0)=0=u(o)=0 .
\end{gathered}
$$

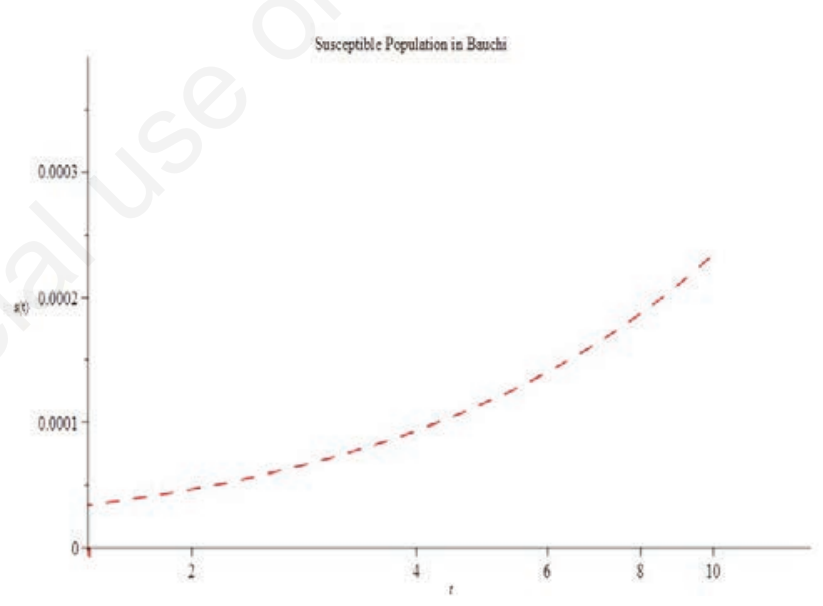

Figure 2. Graph of Susceptible population in Bauchi state.

Table 1. Derived model parameters.

\begin{tabular}{lcc} 
Parameters & Values & Source \\
$\alpha$ & 0.000001133 & BSMOH (2020) \\
$\gamma$ & 1.000000 & Worked \\
$\varphi$ & 0.00000187 & Worked \\
$\delta$ & 14 days & WHO (2020) \\
\hline d & 0.000929 & NBS (2020) \\
N & $7,468,016$ & NPC (2020) \\
\hline$\varepsilon$ & 0.631 & Worked \\
q & 0.000000536 & Worked \\
\hline m & $4 / 14$ & BSMOH (2020) \\
$\beta$ & $0.1375 \times 10-5$ & Worked \\
\hline so & 0 & Worked \\
io & 0 & Worked \\
\hline eo & 0 & Worked \\
ro & 0 & Worked \\
\hline qo & 0 & Worked \\
$\mathbf{T}$ & 0.632 & Worked \\
\hline
\end{tabular}


This explains that at diseases free equilibrium, no one was infected.

\section{Recovered class model solution}

$$
\begin{gathered}
\frac{d r}{d t}=d r^{2}-(\varepsilon+d) r-\beta r+\delta e-\frac{\alpha r s}{N}+(\alpha-\delta) r e+(\alpha+\tau+d) r i-(d+m) q r \\
\int \frac{d r}{d t} d t=\int\left[d r^{2}-(\delta+d) r-\beta r+\delta e-\frac{\alpha r s}{N}+(\alpha-\delta) r e+(\alpha+\tau+d) r i-(d+m) q r\right] d t \\
r(t)=d r^{2} t-(\varepsilon+d) r t-\beta r t+\delta e t-\frac{\alpha r s}{N} t+(\alpha-\delta) r e t+(\alpha+\tau+d) r i t-(d+m) q r t+y(t) \\
r(0)=0=y(0)=0
\end{gathered}
$$

\section{Quarantined class model solution}

$$
\begin{gathered}
\frac{d q}{d t}=(d+m) q^{2}-(d+m) q-\beta q+\theta-\frac{\alpha q s}{N}+(\alpha-\delta) e q+(\alpha+\tau+d) i q+d r q \\
\int \frac{d q}{d t} d t=\int\left[(d+m) q^{2}-(d+m) q-\beta q+\sigma r-\frac{\alpha q s}{N}+(\alpha-\delta) e q+(\alpha+\tau+d) i q+d r q\right] d t \\
q\left((t)=(d+m) q^{2} t-(d+m) q t-\beta q t+\theta t-\frac{\alpha q s}{N} t+(\alpha-\delta) e q t+(\alpha+\tau+d) i q t+d r q t+m(t)\right.
\end{gathered}
$$

$q(0)=0=w(0)=0$

\section{Discussion}

From Figures 2 to 5 the graphical representation of every model is shown. The values used in the models are provided in Table 1. As seen in Figure 2 as soon as the disease was recorded in Bauchi, the number of susceptible people increased rapidly as the graph raises up away from the horizontal axis. Figure 4 shows the infection is increasing exponentially, as reported in previous literature by Lian et al. ${ }^{12}$

Again the models shows there is a high possibility of decline in infection rate because the ratio between the incidence rate and rate of population quarantined is

$$
R_{o}=0.0000026<1 R_{o}=\left(\frac{\varphi}{\tau+\alpha}\right)<1=2.6 \times 10^{-6}<1 .
$$

The basic reproductive number $R_{o}=0.0000026<1$, which is close to zero can be possibly made zero if quarantine regulations are strictly adhered to. Studies have consistently found the major determining factor in containing an out-break is the low reproduction number. ${ }^{13}$

In Figure 6, which is apparently the most important graph of our models, shows the relationship between infected and quarantined class. As the graph presents, the dash line stands for quarantined and the solid blue line represents infected class. Previous studies have documented the effect of quarantine on the spread of COID-19 infection. ${ }^{14}$ Until the quarantine regulations are taken seriously to the point where the dash line moves upward to meet the blue line, the infection will soar. The dash line is raising but not as rapid as the infection rate. The rate of quarantine must be increased.

\section{Conclusions}

Contact tracing must be taken seriously because the models

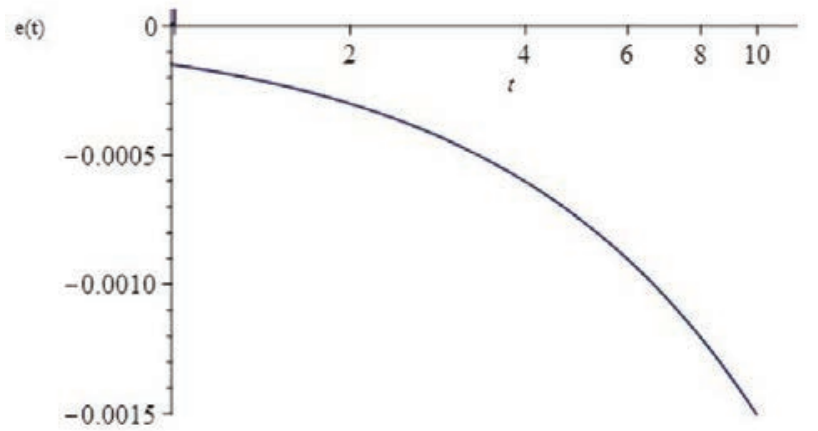

Figure 3. Graph of exposed population in Bauchi state.

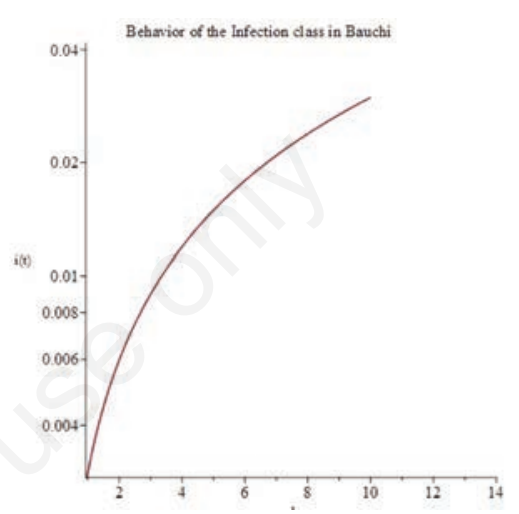

Figure 4. Graph of infected class in Bauchi state.

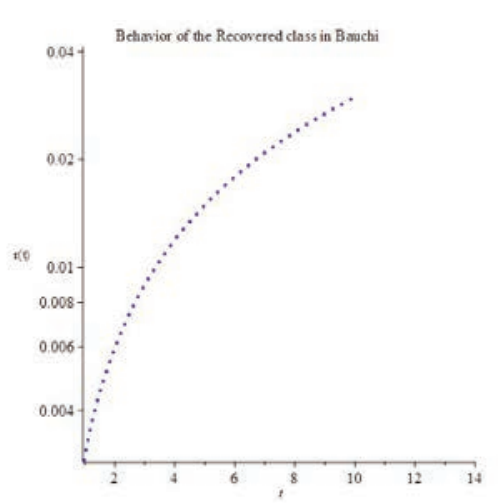

Figure 5. Graph of recovered population in Bauchi state.

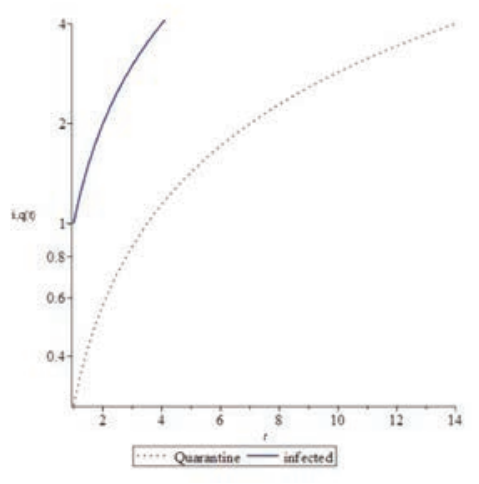

Figure 6. Graph of effect of quarantine on infected population. 
show the rise in infected class is a sign of high vulnerability of the population and lack of vaccine. Unless quarantine is done adequately, despite high rate of recovery, the rate of infection will keep increasing in the absence of the COVID-19 vaccine at the moment.

\section{Limitations of this study}

The models we developed in this study are only true to the extent of the validity of the information at our disposal at the time of the study. However, the COVID-19 pandemic is still ongoing and the infection matrix is still emerging. Mathematical models depend heavily on the plausibility of their structure and simplifying assumptions and on the availability of estimates for their (main) parameters. The latter, in turn, limits model complexity as a lack of suitable data argues for a coarser model". ${ }^{15}$

\section{References}

1. Zhongwei J, Zuhong L. Modelling COVID-19 transmission: from data to intervention. Lancet Infect Dis. 2020.

2. Zhang J, Litvinova M, Wang W, Wang Y, Deng X, Chen X, et al. Evolving epidemiology and transmission dynamics of coronavirus disease 2019 outside Hubei province, China: a descriptive and modelling study. Lancet Infect Dis. 2020.

3. Cucinotta D, Vanelli M. WHO Declares COVID-19 a Pandemic. Acta Bio Med 2020;91:157-60.

4. Rothan HA, Byrareddy SN. The epidemiology and pathogenesis of coronavirus disease (COVID-19) outbreak. J Autoimmun 2020.

5. Wu YC, Chen CS, Chan YJ. The outbreak of COVID-19: An overview. J Chin Med Assoc 2020.

6. Wordmeters. COVID-19 CORONAVIRUS PANDEMIC
[Internet]. 2020. Available from: https://www.worldometers.info/coronavirus/

7. NCDC. COVID-19 Nigeria [Internet]. COVID-19 Nigeria Update. 2020. Available from: https://covid19.ncdc.gov.ng/

8. Bauchi MOH. COVID-19 Update, EOC, Bauchi State MOH. 2020.

9. Butcher J. Runge-Kutta methods for ordinary differential equations [Internet]. Runge-Kutta methods for ordinary differential equations. 2005 [cited 2020 May 15]. p. 1-248. Available from: https://www.math.auckland.ac.nz/ butcher/CONFERENCES/JAPAN/KYUSHU/kyushu-slides.pdf

10. Diekmann O, Heesterbeek JAP, Metz JAJ. On the definition and the computation of the basic reproduction ratio $\mathrm{R} 0$ in models for infectious diseases in heterogeneous populations. J Math Biol 1990;28:365-82.

11. Diekmann O, Heesterbeek JAP, Roberts MG. The construction of next-generation matrices for compartmental epidemic models. J R Soc Interface 2010;7:873-85.

12. Liang K. Mathematical model of infection kinetics and its analysis for COVID-19, SARS and MERS. Infect Genet Evol. 2020.

13. Tuite AR, Fisman DN, Greer AL. Mathematical modelling of COVID-19 transmission and mitigation strategies in the population of Ontario, Canada. Can Med Assoc J 2020.

14. Mandal S, Bhatnagar T, Arinaminpathy N, Agarwal A, Chowdhury A, Murhekar M, et al. Prudent public health intervention strategies to control the coronavirus disease 2019 transmission in India: A mathematical model-based approach. Indian J Med Res 2020.

15. Grundmann H, Hellriegel B. Mathematical modelling: A tool for hospital infection control. Lancet Infect Dis 2006. 